\title{
The Relationship between Standard Automated Perimetry and GDx VCC Measurements
}

\author{
Nicolaas J. Reus and Hans G. Lemij
}

Purpose. To investigate the relationship between retinal light sensitivity measured with standard automated perimetry (SAP) and retardation of the peripapillary retinal nerve fiber layer (RNFL) measured with the GDx VCC (Laser Diagnostic Technologies, Inc., San Diego, CA).

Methods. Forty-seven healthy subjects and 101 patients with glaucoma were examined with SAP and with the commercially available scanning laser polarimeter GDx VCC, with automated individualized compensation of anterior segment birefringence. Individual visual field test points and peripapillary RNFL retardation measurements were grouped into six corresponding sectors. The correlation between perimetry and GDx VCC measurements was determined, and the relationship between RNFL retardation and perimetry, expressed both in the standard decibel scale and in an unlogged scale, was described with linear regression analysis.

RESULTS. A statistically significant correlation was found in most sectors between perimetry and GDx VCC measurements in patients with glaucoma, but not in healthy subjects. A linear relationship was found between the unlogged sensitivities and GDx VCC measurements for the superotemporal and inferotemporal sectors. In the decibel scale, this relationship was curvilinear.

Conclusions. GDx VCC measurements of the peripapillary RNFL relate well with functional loss in glaucoma. Based on the observed relationships between function and structure, patients with mild to moderate visual field loss in glaucoma may be better monitored with the GDx VCC and patients who have severe loss, with SAP. (Invest Ophthalmol Vis Sci. 2004;45: 840 - 845) DOI:10.1167/iovs.03-0646

G laucoma is an optic neuropathy with loss of retinal ganglion cells (RGCs) and their axons. ${ }^{1-3}$ The loss of RGC axons may be apparent structurally as a local and/or a diffuse thinning of the retinal nerve fiber layer $(\mathrm{RNFL})^{3-6}$ and of the neuroretinal rim. ${ }^{5}$ Functionally, RGC atrophy leads to characteristic visual field defects. ${ }^{7}$ In clinical practice, as well as in clinical trials, both structural and functional losses are assessed for the diagnosis and monitoring of glaucoma. ${ }^{1,8,9}$

Functional losses by glaucoma are traditionally evaluated with standard automated perimetry (SAP). Perimetry assesses

From the Glaucoma Service, The Rotterdam Eye Hospital, Rotterdam, The Netherlands.

Presented in part at the annual meeting of the Association for Research in Vision and Ophthalmology, Fort Lauderdale, Florida, May 2003.

Supported by The Rotterdam Eye Hospital Research Foundation; Stichting Glaucoomfonds, Oegstgeest, The Netherlands; and a GDx VCC on loan from Laser Diagnostic Technologies, Inc.

Submitted for publication June 24, 2003; revised October 9 and November 26, 2003; accepted December 8, 2003.

Disclosure: N.J. Reus, Laser Diagnostic Technologies, Inc. (F); H.G. Lemij, Laser Diagnostic Technologies, Inc. (F, C)

The publication costs of this article were defrayed in part by page charge payment. This article must therefore be marked "advertisement" in accordance with 18 U.S.C. $\$ 1734$ solely to indicate this fact.

Corresponding author: Nicolaas J. Reus, Glaucoma Service, The Rotterdam Eye Hospital. PO Box 70030, NL-3000 LM Rotterdam, The Netherlands; reus@oogziekenhuis.nl. the differential light sensitivity (unlogged-DLS $=L_{b} /\left(L_{t}-L_{b}\right)$, where $L_{b}$ is background luminance and $L_{t}$ the stimulus luminance at threshold) ${ }^{10}$ at various locations in the central retina which is typically expressed in a decibel scale (decibel-DLS = $10 \cdot \log _{10} L_{\max } /\left(L_{t}-L_{b}\right)$ where $L_{\max }$ is the perimeter's maximum stimulus luminance). The relationship between function and structure has been found to be curvilinear for the relationships between decibel-DLS and number of ganglion cells ${ }^{1,12}$ and neuroretinal rim area. ${ }^{13-15}$ However, when differential light sensitivity is expressed in the unlogged-DLS scale, function appears to relate linearly to structure, as has been shown by Garway-Heath et al. ${ }^{11,13,16}$

Structural losses of the RNFL can be evaluated with scanning laser polarimetry (SLP). Instruments featuring this technique, such as the GDx nerve fiber analyzer (NFA) and the GDx VCC (both from Laser Diagnostic Technologies, Inc., San Diego, CA), estimate the thickness of the RNFL by measuring the summed retardation of a polarized scanning laser beam, induced by the form-birefringent microtubules that support the RGC axons. ${ }^{17-19}$ Retardation in these instruments is usually expressed in micrometers of thickness, based on the relationship between the amount of retardation and the histologically determined RNFL thickness in monkey eyes, ${ }^{19}$ although this relationship may vary somewhat in each nerve fiber bundle around the optic nerve head (Huang X, et al. IOVS 2003;44: ARVO E-Abstract 3363).

Both the GDx NFA and the GDx VCC are equipped with an anterior segment compensator to cancel the birefringent effects of the cornea and, to a lesser degree, the lens. Whereas the compensator of the GDx NFA is fixed, the GDx VCC is equipped with an automated so-called variable corneal compensator (VCC), allowing eye-specific compensation of anterior segment birefringence. Because of large interindividual and intraindividual variability in anterior segment birefringence, ${ }^{20-22}$ measurements with the GDx NFA do not always accurately reflect the $\mathrm{RNFL}^{23}$ and have been reported to have only a moderate correlation with perimetry. ${ }^{24-30}$ Equipped with a VCC, SLP has been shown to allow objective assessment of localized structural RNFL defects. ${ }^{23}$ In addition, using a modified GDx NFA, Bowd et al. ${ }^{24}$ have shown that SLP measurements with VCC in patients with predominantly mild glaucomatous damage correlate better with perimetry than those with fixed compensation.

The purpose of the present study was to investigate the functional-structural relationship between standard automated perimetry and measurements of peripapillary RNFL retardation with the commercially available GDx VCC in healthy subjects and patients with glaucoma.

\section{Methods}

Forty-seven healthy subjects and 101 patients with glaucoma were examined with SAP (Humphrey Field Analyzer [HFA]II, 24-2 FullThreshold or Swedish interactive threshold algorithm [SITA] Standard test program; Carl Zeiss Meditec, Dublin, CA) and SLP with individualized compensation of anterior segment birefringence (GDx VCC; Laser Diagnostic Technologies, Inc.). The research adhered to the tenets of the Declaration of Helsinki. Informed consent was obtained from the subjects after explanation of the nature and possible conse- 
quences of the study. The institutional human experimentation committee had approved the research.

Patients with glaucoma were recruited consecutively from an ongoing longitudinal follow-up study $(n=96)$ or after referral by a glaucoma specialist (HGL) for clinical reasons $(n=5)$. All patients with glaucoma had a reproducible glaucomatous visual field defect with SAP and a glaucomatous appearance of the optic disc. Only one eye per patient was used for analysis. If more than one eye was eligible, the one with the more positive mean deviation (MD) for SAP was used. All patients with glaucoma were of white ethnic origin and had a visual acuity of 20/40 or better. Patients with any significant coexisting ocular disease, including posterior segment eye diseases and corneal diseases, or systemic diseases with possible ocular involvement, such as diabetes mellitus, were excluded.

Healthy subjects were recruited either consecutively from an ongoing longitudinal follow-up study or from employees of the Rotterdam Eye Hospital and their spouses and friends. All healthy subjects had a Glaucoma Hemifield Test result of "within normal limits" for SAP, healthy-looking optic discs, and an intraocular pressure of $21 \mathrm{~mm} \mathrm{Hg}$ or less, measured with Goldmann applanation tonometry. All subjects were of white ethnic origin and had a visual acuity of 20/40 or better. None had a significant history of ocular disease, including posterior segment eye diseases and corneal diseases, relatives in the first and/or second degree with glaucoma, systemic hypertension for which medication was used, diabetes mellitus, or any other systemic disease.

For SAP, appropriate near refractive correction was used. Reliability criteria applied were: (1) fixation losses less than or equal to $25 \%$ and (2) false-positive and false-negative response rates less than or equal to $20 \%$ for the Full Threshold test paradigm and less than or equal to $7 \%$ for the SITA-Standard test paradigm. In the patients with glaucoma, however, higher false-negative response rates were accepted. The mean period between perimetry and GDx VCC measurements was $0.8 \pm 2.5$ weeks (SD; range, $0-12$ ) and $12.2 \pm 11.0$ weeks (range, $0-27)$ in patients with glaucoma and in healthy subjects, respectively.

The mean MD was $-9.39 \pm 7.45 \mathrm{~dB}(\mathrm{SD})$ and $0.48 \pm 1.22 \mathrm{~dB}$ for patients with glaucoma and healthy subjects, respectively. The mean age of the patients with glaucoma and the healthy subjects was $62 \pm$ 10 years and $59 \pm 13$ years, respectively, which was not significantly different (two-sample $t$-test, $P=0.10$ ).

In the glaucoma group, 54 of the 101 subjects (54\%) were men. Of the healthy subjects, 23 of the 47 (49\%) were men. Fifty-two of the 101 eyes $(51 \%)$ in the glaucoma group were right eyes; in the healthy group, 22 of the 47 eyes ( $47 \%$ ) were right eyes.

In all subjects, both eyes were scanned with the GDx VCC, starting with the right eye. The spherical equivalent refractive error of each eye was entered into the software to allow the GDx VCC to focus properly on the retina. The patient's face was gently placed into the face mask of the GDx VCC. To maintain the same orientation of the slow axes of the birefringent structures in the eye to that of the instrument's compensator, the operator assisted patients in keeping their heads as vertical as possible during all measurements. The pupils of the patients were undilated, and the room lights were left on. For each scan, the operator aligned the instrument with the cornea and the sclera of the measured eye

First, anterior segment birefringence was assessed for each eye of each subject with the method described by Zhou and Weinreb. ${ }^{31}$ To this end, the magnitude of the compensator of the GDx VCC was automatically set to zero, after which the fundus was scanned. The interaction between the birefringence of the radially oriented axons of the photoreceptors that constitute Henle's fiber layer in the macula and the anterior segment birefringence resulted in a bow-tie pattern on the retardation image. A dedicated algorithm, incorporated into the GDx VCC software, determined the anterior segment birefringence (consisting of magnitude and axis) from this profile.

The software then used these calculations to automatically adjust the anterior segment compensator to each individual eye, and both eyes were scanned again with individualized compensation. Adequate compensation of anterior segment birefringence was verified subjec-

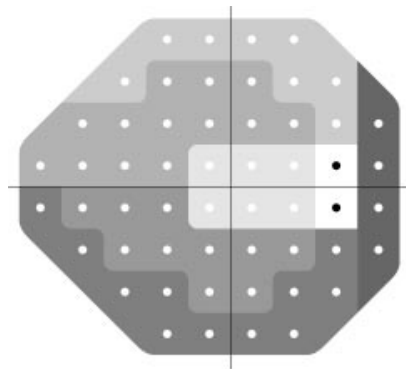

HFA

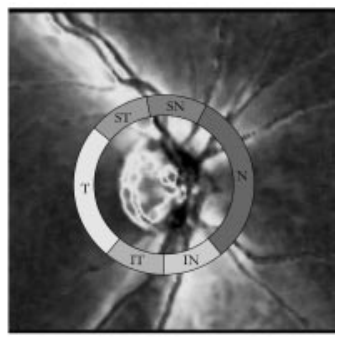

GDx VCC
Figure 1. Test pattern of the HFA 24-2 (Carl Zeiss Meditec) paradigm for a right eye (left) and a GDx VCC (Laser Diagnostic Technologies, Inc.) retardation image of a right eye (right), with a measurement circle superimposed. In the present study, visual field test points and peripapillary GDx VCC measurements were grouped in corresponding sectors, as suggested by Garway-Heath et al. ${ }^{32}$ Corresponding sectors were grayscaled and named after the position of the sector in the GDx VCC image in relation to the optic disc.

tively by looking at the retardation pattern in the macular region that had to be uniformly weak with a cross- or donut-shaped pattern. The typical time to measure both eyes of a patient was 3 minutes. All scans had to be of high quality - that is, with a centered optic disc, well focused, even and just illuminated throughout the image, and without any motion artifacts. In addition, the measurements had to pass the five scan-quality checks that are automatically performed by the GDx VCC software.

The margin of the optic disc was manually marked with an ellipse on a reflection image of the fundus. The GDx VCC software positioned a circle, 8 pixels wide ( $\sim 0.4 \mathrm{~mm}$ in an emmetropic eye) and with an inner diameter of 54 pixels $(\sim 2.5 \mathrm{~mm}$ in an emmetropic eye), centered on the center of the ellipse. The instrument processed the retardation values within this band to give 256 values evenly distributed along the circle. These values were subsequently grouped into 64 sectors and exported by the software.

The relationship between visual field test points and regions of the optic disc as described by Garway-Heath et al. ${ }^{32}$ was used to correlate the visual fields to the GDx VCC measurements. The 64 peripapillary sectors in the GDx VCC retardation image and the 52 visual field test points were grouped into six corresponding sectors (Fig. 1). Because of the fixed dimensions of the exported sectors in the GDx VCC, the size and orientation of the six optic nerve head sectors differed slightly from those presented by Garway-Heath et al., but were consistent with their published relationship between optic nerve head location and visual field test points. ${ }^{32}$ The peripapillary measurement circle was divided into one $90^{\circ}$ sector (temporal [T]), one $112.5^{\circ}$ sector (nasal $[\mathrm{N}]$ ), and four equally sized sectors of approximately $39.4^{\circ}$ (superotemporal $[\mathrm{ST}]$, superonasal $[\mathrm{SN}]$, inferotemporal [IT], and inferonasal [IN]). The average retardation was calculated for each sector.

The differential light sensitivity in each visual field sector was also averaged and expressed in both the typically used decibel scale (decibel-DLS) and in the unlogged DLS scale (unlogged-DLS $=L_{b} /\left(L_{t}-L_{b}\right)$ $\left.=\left(L_{b} / L_{\max }\right) \cdot L_{\max } /\left(L_{t}-L_{b}\right)=\left(L_{b} / L_{\max }\right) \cdot 10^{D L S_{d B} / 10}\right)$. For the HFA, $L_{b}$ $=31.6 \mathrm{asb}$ and $L_{\max }=10,000 \mathrm{asb}$.

To measure the degree of association between SAP and GDx VCC measurements, we calculated Spearman's rank correlation coefficient $\left(r_{\mathrm{S}}\right)$ for each sector. Subsequently, the relationship between perimetry expressed in both the decibel-DLS and the unlogged-DLS scale and GDx VCC measurements was described with linear regression analysis.

\section{Results}

The relationship between perimetry and GDx VCC measurements is graphically presented for all sectors in Figure 2. We found statistically significant correlations between standard 
A

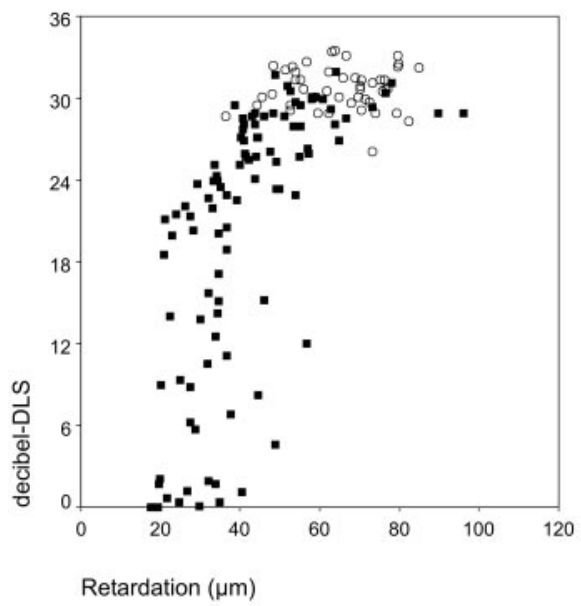

B

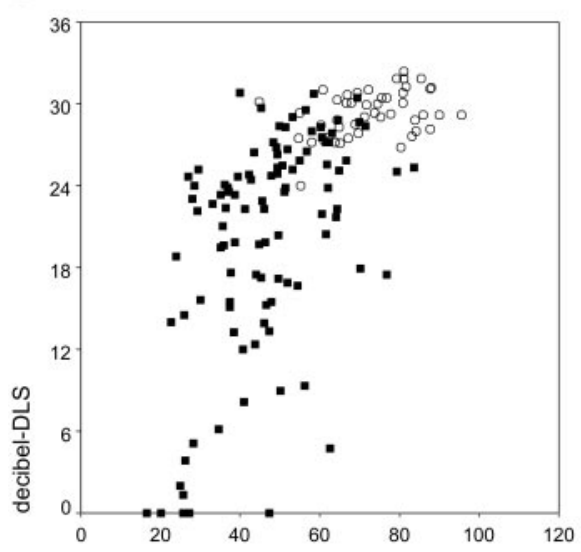

Retardation $(\mu \mathrm{m})$

C

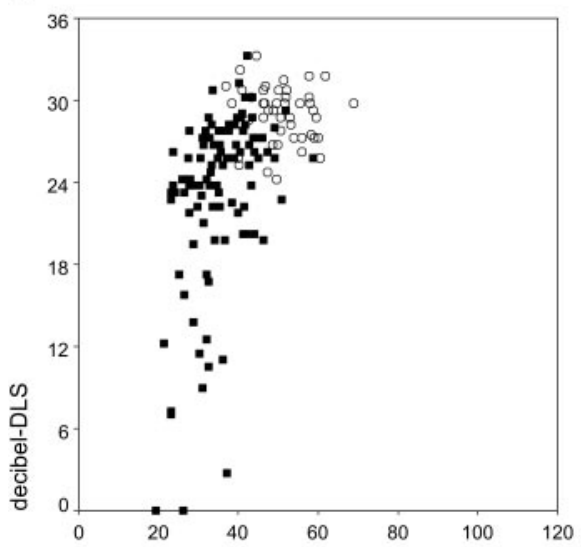

Retardation $(\mu \mathrm{m})$
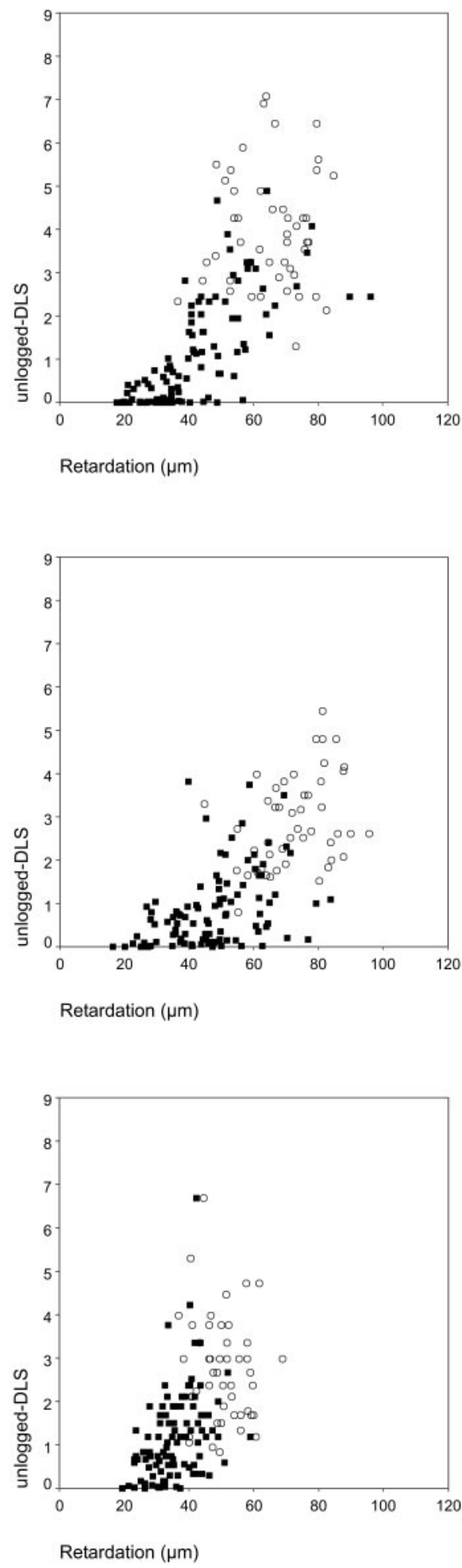

Figure 2. Scatterplots of differential light sensitivity (DLS), expressed as decibel-DLS (left) and unloggedDLS (right), against peripapillary RNFL retardation measured with the GDx VCC (Laser Diagnostic Technologies, Inc.). ( $\bigcirc)$ Healthy subjects; (ם) patients with glaucoma. Sectors: (A) superotemporal; (B) superonasal; (C) nasal; (D) inferonasal; (E) inferotemporal; (F) temporal. automated perimetry and GDx VCC measurements in patients with glaucoma $(P<0.001)$, in all sectors except the temporal one $(P=0.059)$, with $r_{\mathrm{S}}$ of $0.77,0.52,0.46,0.51,0.38$, and 0.19 for the sectors ST, SN, N, IN, IT, and T, respectively (Fig. 2 , Table 1 ). In healthy subjects, no statistically significant correlations between perimetry and GDx VCC measurements were found in any sector $(P>0.13)$, except the superonasal one $(P=0.012$; Fig. 2 , Table 1$)$.
When fit with a least-squares linear regression model, the relationship between decibel-DLS and RNFL retardation in healthy subjects and patients with glaucoma yielded $R^{2}$ values of $0.48,0.42,0.29,0.37$, and 0.35 for the sectors ST, SN, N, IN, and IT, respectively (for slopes, $P<0.001$ ). For the unlogged-DLS, the $R^{2}$ values of the linear regression models were $0.52,0.48,0.26,0.35$, and 0.43 , respectively ( $P$ of slopes $<0.001)$. 
D

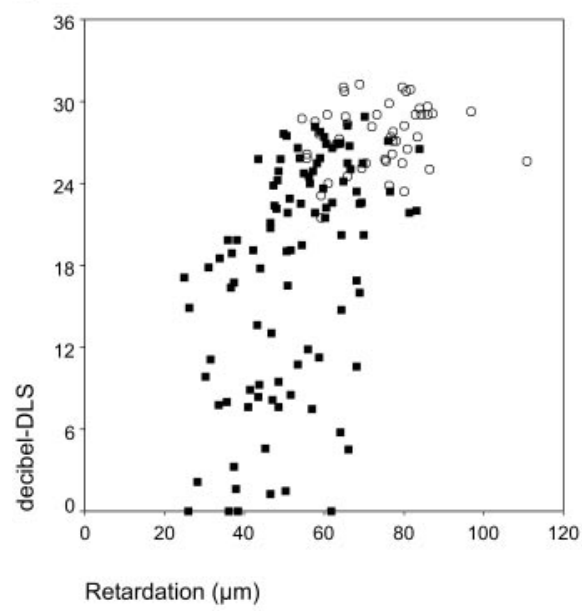

E

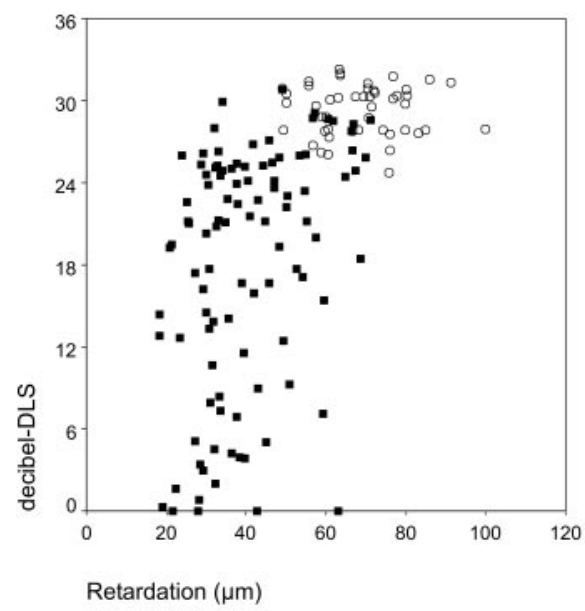

$\mathbf{F}$

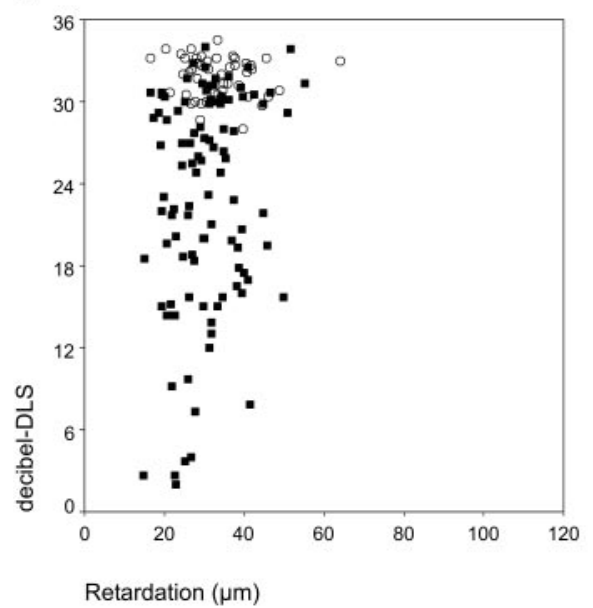

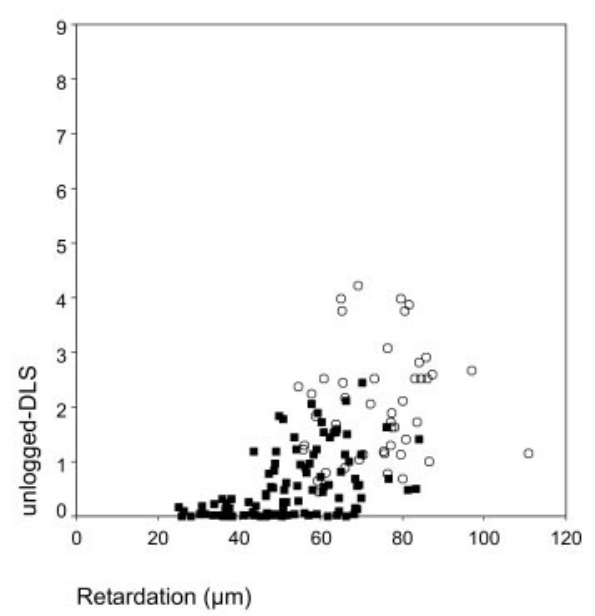
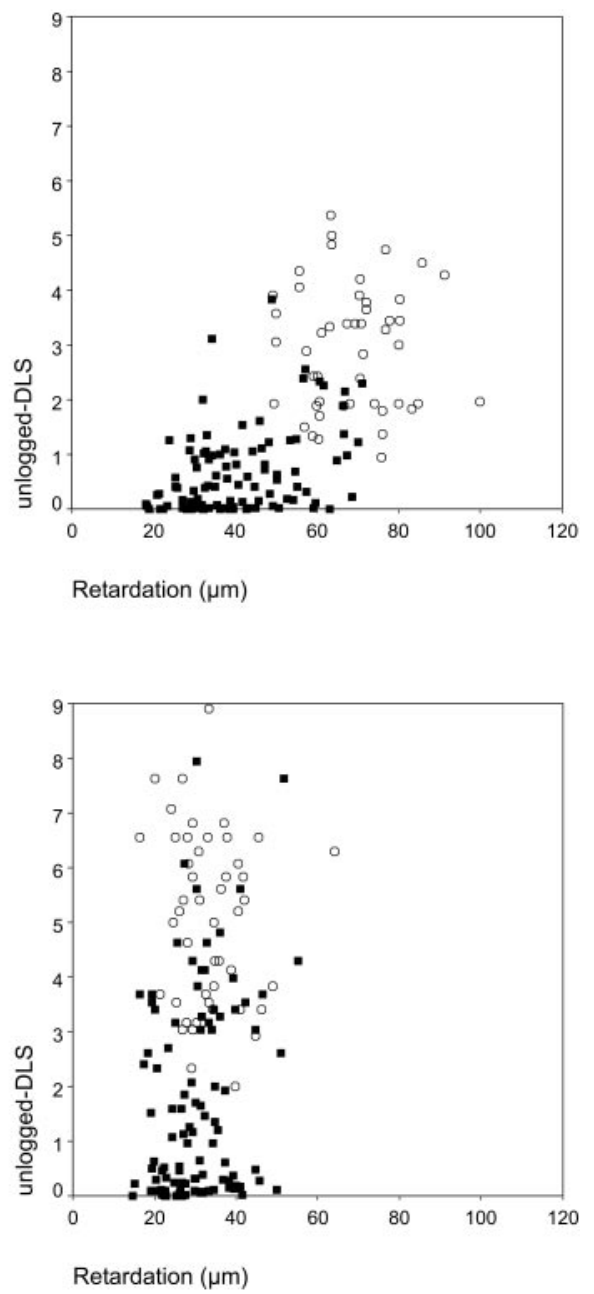

Figure 2. (Continued)

For the sectors ST, SN, and IT, linear regression analysis yielded statistically significant better fits for the unlogged-DLS scale than for the decibel-DLS scale (signed rank test, $P=$ $0.011, P<0.001$, and $P=0.011$, respectively). Conversely, for the sectors $\mathrm{N}$ and IN, linear regression analysis yielded statistically significant better fits for the decibel-DLS scale (signed rank test, $P<0.001$ and $P=0.004$, respectively). We did not fit the relationship between perimetry and GDx VCC measure- ments for the temporal sector with linear regression analysis, because they did not correlate.

\section{Discussion}

We have shown a correlation between standard automated perimetry and GDx VCC measurements in patients with glau- 
TABLE 1. Correlation between SAP and GDx VCC Measurements

\begin{tabular}{lccccc}
\hline & \multicolumn{2}{c}{$\begin{array}{c}\text { Glaucoma } \\
\text { Patients }\end{array}$} & & \multicolumn{2}{c}{$\begin{array}{c}\text { Healthy } \\
\text { Subjects }\end{array}$} \\
\cline { 2 - 5 } \multicolumn{1}{c}{ Sector } & $\boldsymbol{r}_{\mathbf{s}}$ & $\boldsymbol{P}$ & & $\boldsymbol{r}_{\mathbf{s}}$ & $\boldsymbol{P}$ \\
\cline { 3 - 6 } Superotemporal & 0.77 & $<0.001$ & & 0.04 & 0.78 \\
Superonasal & 0.52 & $<0.001$ & & 0.37 & 0.012 \\
Nasal & 0.46 & $<0.001$ & & -0.11 & 0.47 \\
Inferonasal & 0.51 & $<0.001$ & & 0.22 & 0.13 \\
Inferotemporal & 0.38 & $<0.001$ & & 0.05 & 0.77 \\
Temporal & 0.19 & 0.059 & & -0.16 & 0.29 \\
\hline
\end{tabular}

Degrees of association, measured with Spearman's rank correlation coefficient $\left(r_{\mathrm{s}} ; P\right)$, between SAP differential light sensitivity and GDx VCC measurements in patients with glaucoma $(n=101)$ and healthy subjects $(n=47)$ for the six sectors described in Figure 1 .

coma, suggesting that GDx VCC measurements relate well with functional loss in glaucoma. However, in healthy subjects, we found virtually no correlation between perimetry and GDx VCC measurements.

For the sectors ST, IT, and SN, the relationship between function and structure was linear in the unlogged-DLS scale. In the standard decibel-DLS scale, a curvilinear relationship was apparent. Because the sectors ST and IT are reportedly most affected by glaucoma ${ }^{3-5,33}$ and also because they represent areas of the visual field close to fixation, we think that the differences in linearity in these sectors are clinically important. Figures $2 \mathrm{~A}$ and $2 \mathrm{E}$, relating to these sectors, show that large differences in GDx VCC measurements correlated with only small differences in decibel-DLS in areas with no to moderate functional loss. In more advanced functional loss, the opposite was true. Therefore, SLP appears to be more suited than SAP for monitoring both healthy subjects at risk of contracting glaucoma and patients with glaucoma with mild to moderate functional damage. In more advanced glaucoma, however, SAP may be more useful than SLP for follow-up. Patients with glaucomatous visual field defects of mixed severity may be best monitored with a combination of SLP and SAP.

This relationship was similar in the SN sector (Fig. 2B) but less pronounced in the $\mathrm{N}$ sector (Fig. 2C). In the IN sector, however, GDx VCC measurements did not appear to be better at detecting mild glaucomatous loss than perimetry (Fig. 2D). We argue, however, that the IN sector, which relates to the uppermost visual field of the HFA 24-2 program, has poor perimetric reproducibility, ${ }^{34}$ which limits its clinical usefulness.

The linear relationship that we found between function (unlogged-DLS) and structure was similar to those reported by Garway-Heath et al. ${ }^{11}$ between unlogged-DLS and the number of RGCs and neuroretinal rim area, ${ }^{16}$ and to the theoretically modeled one by Swanson et al. (Swanson WH, et al. IOVS 2003;44:ARVO E-Abstract 57) between unlogged-DLS and the number of RGCs. In addition, our finding of a curvilinear functional-structural relationship between decibel-DLS and RNFL retardation corresponds with the reported relationships between decibel-DLS and number of RGCs ${ }^{11,12}$ and between decibel-DLS and neuroretinal rim area. ${ }^{14,15}$

Several investigators also studied the relationship between SAP and SLP measurements and reported no correlation or only a mild one in healthy and glaucomatous eyes. ${ }^{24-30}$ Their poor correlation may be attributable to differences in study populations and the use of different parameters. More important, they used SLP with a fixed compensator of anterior segment birefringence, instead of a variable one. Knighton et al. ${ }^{35}$ reasoned that an individualized anterior segment compensation of birefringence would be necessary for accurate measurement of RNFL retardation. ${ }^{20,22}$ Our results support those of Bowd et al., ${ }^{24}$ who compared a variable with a fixed anterior segment compensator and found an improved relationship between visual function and structure with VCC. Of interest, Bowd et al. ${ }^{24}$ found that the relationship between decibel-DLS and GDx measurements with VCC was better described by a linear model than by a curvilinear one in all sectors. However, their data related to predominantly mild glaucomatous damage (mean MD, $-2.74 \pm 3.71 \mathrm{~dB}$; [SD]), whereas we used a much larger range of glaucomatous eyes and also many healthy eyes. Their smaller range may have precluded the detection of curvilinearity in the relationship between function and structure.

In the sectors ST and IT, the $R^{2}$ values of the linear regression models describing the relationship between unlogged-DLS and RNFL retardation were 0.52 and 0.43 , respectively. Therefore, $48 \%$ to $57 \%$ of the variation in unlogged-DLS was not explained by RNFL retardation alone. In the other sectors, $52 \%$ to $95 \%$ of the variation in this relationship was unexplained. Some of this scatter may be due to retardation originating from axons that had their origin outside the points tested by the HFA. Such axons may relate to areas either between the tested points or outside the entire test area displayed in Figure 1. In addition, mismatching of the six optic nerve head (ONH) sectors and the visual field test points in the map constructed by Garway-Heath et al. ${ }^{32}$ may have added to the variation in the correlation between perimetry and GDx VCC measurements. Garway-Heath et al. ${ }^{32}$ have reported that the range of possible positions at the $\mathrm{ONH}$ of $\mathrm{RGC}$ axons originating from each visual field test point location covers almost $30^{\circ}$. Factors that contributed to the variation in that study were the intereye variability in the position of the $\mathrm{ONH}$ in relation to the fovea, intereye variability in retinal magnification, and variations in shape, rotation, and tilt of the $\mathrm{ONH}^{32}$ Apart from these variations, our data may also have been influenced by variation in the positioning of the head during SLP. Some of the unexplained variation in the relationship between DLS and RNFL retardation may also be attributable to the reproducibility of measurements with SAP and SLP. For example, the variability in DLS within subjects has been shown to be substantial. ${ }^{34,36,37}$ Therefore, combining the results of several subsequent visual field tests may improve the relationship between DLS and RNFL retardation. To what extent the variability of GDx VCC measurements has influenced our results is unclear, because its reproducibility of measurements has not yet been assessed. Some variation in DLS may also have been due to age-related changes in the ocular media as well as age-related changes of the retina, ${ }^{38}$ other than loss of RGCs, and changes in the central nervous system.

For DLS values near zero, we still measured retardation equivalent to approximately $20 \mu \mathrm{m}$ or more (Fig. 2). A possible explanation for this offset is that some RGCs had stopped functioning, but their axons were still present, thus exhibiting birefringence. Axons have been identified in the RNFL that have no demonstrable visual function. ${ }^{39}$ Another explanation is that we measured residual retardation from incomplete compensation of anterior segment birefringence or that we measured retardation induced by birefringent structures in the eye other than the RGC axons or anterior segment, as has been suggested by measurements with polarization sensitive optical coherence tomography (De Boer JF, et al. IOVS 2003; 44:ARVO E-Abstract 3388). It is unclear whether an offset may have been present in the instrument itself. The offset may also have been caused by the retardation originating from axons that had their origin outside the tested points of the HFA 24-2 program (i.e., either between the testing points or outside the tested central $24^{\circ}$ area).

In the present study, we found a linear relationship between unlogged-DLS and RNFL retardation and a curvilinear relationship between decibel-DLS and RNFL retardation. This suggests that the unlogged-DLS scale may be more appropriate 
for comparing structural and functional measurements than the standard $\mathrm{dB}$ scale, as suggested earlier by Garway-Heath et al. ${ }^{13}$ Clinically, however, the standard decibel scale may be more appropriate, because the variability of perimetric measurements between healthy subjects appears to be less when expressed in the decibel-DLS scale than in the unlogged-DLS scale (cf. Figs. 2A-F; right and left images). This apparently improved variability may, however, lower its sensitivity to detecting change, notably at the higher end of the decibel-DLS scale. Such change might, as stated earlier, be better monitored with SLP than with SAP.

\section{Acknowledgments}

The authors thank Paul G. H. Mulder, PhD (Department of Epidemiology \& Biostatistics, Erasmus MC, Rotterdam, The Netherlands) for valuable discussions.

\section{References}

1. American Academy of Ophthalmology. Preferred Practice Pattern. Primary Open Angle Glaucoma. San Francisco, CA: American Academy of Ophthalmology; 2000.

2. Kerrigan-Baumrind LA, Quigley HA, Pease ME, Kerrigan DF, Mitchell RS. Number of ganglion cells in glaucoma eyes compared with threshold visual field tests in the same persons. Invest Ophthalmol Vis Sci. 2000;41:741-748.

3. Quigley HA, Addicks EM, Green WR. Optic nerve damage in human glaucoma. III. Quantitative correlation of nerve fiber loss and visual field defect in glaucoma, ischemic neuropathy, papilledema, and toxic neuropathy. Arch Ophthalmol. 1982;100:135146.

4. Jonas JB, Schiro D. Localised wedge shaped defects of the retinal nerve fibre layer in glaucoma. Br J Ophthalmol. 1994;78:285-290.

5. Tuulonen A, Airaksinen PJ. Initial glaucomatous optic disk and retinal nerve fiber layer abnormalities and their progression. $A m J$ Ophthalmol. 1991;111:485-490.

6. Quigley HA, Miller NR, George T. Clinical evaluation of nerve fiber layer atrophy as an indicator of glaucomatous optic nerve damage. Arch Ophthalmol. 1980;98:1564-1571.

7. Keltner JL, Johnson CA, Cello KE, et al. Classification of visual field abnormalities in the ocular hypertension treatment study. Arch Ophthalmol. 2003;121:643-650.

8. Heijl A, Leske MC, Bengtsson B, et al. Reduction of intraocular pressure and glaucoma progression: results from the Early Manifest Glaucoma Trial. Arch Ophthalmol. 2002;120:1268-1279.

9. Gordon MO, Kass MA. The Ocular Hypertension Treatment Study: design and baseline description of the participants. Arch Ophthalmol. 1999;117:573-583.

10. Greve EL. Single and multiple stimulus static perimetry in glaucoma: the two phases of perimetry. Thesis. Doc Ophthalmol. 1973;36:1-355

11. Garway-Heath DF, Caprioli J, Fitzke FW, Hitchings RA. Scaling the hill of vision: the physiological relationship between light sensitivity and ganglion cell numbers. Invest Ophthalmol Vis Sci. 2000; 41:1774-1782.

12. Harwerth RS, Carter-Dawson L, Shen F, Smith EL III, Crawford MLJ. Ganglion cell losses underlying visual field defects from experimental glaucoma. Invest Ophthalmol Vis Sci. 1999;40:2242-2250.

13. Garway-Heath DF, Holder GE, Fitzke FW, Hitchings RA. Relationship between electrophysiological, psychophysical, and anatomical measurements in glaucoma. Invest Ophthalmol Vis Sci. 2002; 43:2213-2220.

14. Jonas JB, Grundler AE. Correlation between mean visual field loss and morphometric optic disk variables in the open-angle glaucomas. Am J Ophthalmol. 1997;124:488- 497.

15. Airaksinen PJ, Drance SM, Douglas GR, Schulzer M. Neuroretinal rim areas and visual field indices in glaucoma. Am J Ophthalmol. 1985;99:107-110.

16. Garway-Heath DF, Viswanathan A, Westcott M, et al. Relationship between perimetric light sensitivity and optic disc neuroretinal rim area. In: Wall M, Wild JM, eds. Perimetry Update 1998/1999. The Hague, The Netherlands: Kugler Publications; 1999:381-389.

17. Huang XR, Knighton RW. Linear birefringence of the retinal nerve fiber layer measured in vitro with a multispectral imaging micropolarimeter. J Biomed Opt. 2002;7:199-204.

18. Dreher AW, Bailey ED. Assessment of the retinal nerve fiber layer by scanning-laser polarimetry. SPIE. 1993;1877:266-271.

19. Weinreb RN, Dreher AW, Coleman A, et al. Histopathologic validation of Fourier-ellipsometry measurements of retinal nerve fiber layer thickness. Arch Opbthalmol. 1990;108:557-560.

20. Knighton RW, Huang XR. Linear birefringence of the central human cornea. Invest Ophthalmol Vis Sci. 2002;43:82-86.

21. Weinreb RN, Bowd C, Greenfield DS, Zangwill LM. Measurement of the magnitude and axis of corneal polarization with scanning laser polarimetry. Arch Ophthalmol. 2002;120:901-906.

22. Greenfield DS, Knighton RW, Huang XR. Effect of corneal polarization axis on assessment of retinal nerve fiber layer thickness by scanning laser polarimetry. Am J Ophthalmol. 2000;129:715-722.

23. Reus NJ, Colen TP, Lemij HG. Visualization of localized retinal nerve fiber layer defects with the GDx with individualized and with fixed compensation of anterior segment birefringence. $O p h$ thalmology. 2003;110:1512-1516.

24. Bowd C, Zangwill LM, Weinreb RN. Association between scanning laser polarimetry measurements using variable corneal polarization compensation and visual field sensitivity in glaucomatous eyes. Arch Ophthalmol. 2003;121:961-966.

25. Shields JR, Chen PP, Mills RP. Topographic mapping of glaucomatous visual field defects to scanning laser polarimetry of the peripapillary nerve fiber layer. Ophthalmic Surg Lasers. 2002;33:123126.

26. Kwon YH, Hong S, Honkanen RA, Alward WL. Correlation of automated visual field parameters and peripapillary nerve fiber layer thickness as measured by scanning laser polarimetry. $J$ Glaucoma. 2000;9:281-288.

27. Niessen AG, Van Den Berg TJ, Langerhorst CT, Greve EL. Retinal nerve fiber layer assessment by scanning laser polarimetry and standardized photography. Am J Ophthalmol. 1996;121:484- 493.

28. Chen YY, Chen PP, Xu L, et al. Correlation of peripapillary nerve fiber layer thickness by scanning laser polarimetry with visual field defects in patients with glaucoma. J Glaucoma. 1998;7:312-316.

29. Tjon-Fo-Sang MJ, Lemij HG. The sensitivity and specificity of nerve fiber layer measurements in glaucoma as determined with scanning laser polarimetry. Am J Ophthalmol. 1997;123:62-69.

30. Weinreb RN, Shakiba S, Sample PA, et al. Association between quantitative nerve fiber layer measurement and visual field loss in glaucoma. Am J Ophthalmol. 1995;120:732-738.

31. Zhou Q, Weinreb RN. Individualized compensation of anterior segment birefringence during scanning laser polarimetry. Invest Ophthalmol Vis Sci. 2002;43:2221-2228.

32. Garway-Heath DF, Poinoosawmy D, Fitzke FW, Hitchings RA. Mapping the visual field to the optic disc in normal tension glaucoma eyes. Ophthalmology. 2000;107:1809-1815.

33. Sommer A, Katz J, Quigley HA, et al. Clinically detectable nerve fiber atrophy precedes the onset of glaucomatous field loss. Arch Ophthalmol. 1991;109:77-83.

34. Heijl A, Lindgren G, Olsson J. Normal variability of static perimetric threshold values across the central visual field. Arch Ophthalmol. 1987; 105:1544-1549.

35. Knighton RW, Huang XR, Greenfield DS. Analytical model of scanning laser polarimetry for retinal nerve fiber layer assessment. Invest Ophthalmol Vis Sci. 2002;43:383-392.

36. Heijl A, Lindgren A, Lindgren G. Test-retest variability in glaucomatous visual fields. Am J Ophthalmol. 1989;108:130-135.

37. Keltner JL, Johnson CA, Quigg JM, et al. Confirmation of visual field abnormalities in the Ocular Hypertension Treatment Study. Ocular Hypertension Treatment Study Group. Arch Ophthalmol. 2000; 118:1187-1194.

38. Gao H, Hollyfield JG. Aging of the human retina: differential loss of neurons and retinal pigment epithelial cells. Invest Ophthalmol Vis Sci. 1992;33:1-17.

39. Quigley HA, Dunkelberger GR, Green WR. Retinal ganglion cell atrophy correlated with automated perimetry in human eyes with glaucoma. Am J Ophthalmol. 1989;107:453-464. 